\title{
Access to contraception and sexual and reproductive health information post-abortion: a systematic review of literature from low- and middle-income countries
}

\author{
Claire Rogers, Jaya A R Dantas
}

\begin{abstract}
- Additional material is published online only. To view please visit the journal online (http://dx.doi.org/10.1136/ jfprhc-2016-101469).
\end{abstract}

International Health Programme, School of Nursing, Midwifery and Paramedicine, Faculty of Health Sciences, Curtin University, Perth, Western Australia, Australia

\section{Correspondence to}

Claire Rogers, International Health Programme, School of Nursing, Midwifery and Paramedicine, Faculty of Health Sciences, Curtin University, Bentley Campus, Kent Street, Perth 6102, Western Australia, Australia;

claire.rogers@postgrad.curtin. com.au and

hello@clairerogers.com.au

Received 4 February 2016 Revised 5 January 2017 Accepted 25 January 2017 Published Online First 16 February 2017

\section{CrossMark}

To cite: Rogers C, Dantas JAR. J Fam Plann Reprod Health Care 2017:43:309-318.

\section{ABSTRACT}

Aim This systematic literature review documented, analysed and critiqued the accessibility of contraception and sexual and reproductive health $(\mathrm{SRH})$ information for women living in low- and middle-income countries who have undergone medical or surgical abortion.

Methodology This review systematically collated relevant and recent empirical evidence regarding women's access to contraception and SRH information post-abortion within low- and middle-income countries. The PRISMA (Preferred Reporting Items for Systematic Reviews and Meta-Analyses) framework Guidelines, Flow Diagram and Checklist were utilised to undertake the review. The Ovid (MEDLINE), ProQuest, Science Direct, Web of Science, PUBMED and CINAHL databases were searched and studies that met edibility criteria were assessed for validity and analysis. A narrative synthesis of characteristics and results of the included studies is presented.

Findings After detailed assessment of available and relevant literature, nine studies were selected for inclusion in the review. Studies highlighted barriers to contraception and SRH information including supply limitation, lack of comprehensive education and counselling, lack of skilled post-abortion care (PAC) providers and abortion stigma.

Conclusions The review found that with access to a wide range of contraceptive methods combined with comprehensive SRH information and education, contraception uptake in women post-abortion does increase. The review also highlights the inconsistencies in clinic-reported 'counselling' and what this term actually involves within a PAC setting.
Key message points

- The results of the review highlight that a lack of comprehensive sexual and reproductive health information and education as well as negative provider attitudes are key barriers for women accessing post-abortion contraception in low- and middle-income countries.

- When women are offered a broad range of contraceptive methods and are provided with effective culturally safe contraceptive counselling, the likelihood of them accepting post-abortion contraception increases.

- A clearer understanding of 'counselling' and 'cultural safety and sensitivity' is needed within post-abortion care services to ensure comprehensive information provision and effective support for women.

\section{INTRODUCTION}

\section{Post-abortion care}

Of the 38 million abortions performed annually in low- and middle-income countries, more than half are unsafe. ${ }^{1}{ }^{2}$ Post-abortion care (PAC) is an essential component of comprehensive abortion care (CAC), and refers to a set of interventions designed to respond to the specific needs of women who have miscarried or induced an abortion. ${ }^{3-5}$ The PAC Consortium (2014) states the following five essential elements of PAC necessary for effective and equitable provision of PAC services: 
1 Community and service provider partnerships are vital for prevention of unwanted pregnancies and unsafe abortion; mobilisation of resources to help women receive appropriate and timely care for complications from abortion; and to ensure that health services reflect and meet community expectations and needs.

2 Counselling of women to identify and respond to women's emotional and physical health needs and sexual and reproductive health (SRH) concerns is also a critical component of care.

3 Treatment of incomplete and unsafe abortion and complications that are potentially life-threatening need to be addressed during PAC provision.

4 Contraceptive and family planning services are needed to help women prevent an unwanted pregnancy or practise birth spacing.

5 Reproductive and other health services that are preferably provided on-site or via referrals to accessible and quality facilities in provider' networks are needed to holistically meet women's PAC needs. ${ }^{6} 7$

Even in low- and middle-income countries, such as Nepal and Vietnam, where abortion laws are liberal, unsafe abortion still occurs due to lack of skilled providers, limited access to safe abortion services, and sociocultural and socioeconomic inhibitors. ${ }^{2}$ In countries where abortion is prohibited and illegal and laws are restrictive, such as the Dominican Republic and Sri Lanka, unsafe practices are undertaken and women face an even greater need for accessible, affordable and comprehensive PAC services. $^{2} 89$

The objective of this review was to systematically collate and synthesise recent and relevant research evidence on PAC services provided to women from lowand middle-income countries and their ability to access contraception and SRH information. The findings from this systematic review aim to support global understanding of women's post-abortion experiences relating to access of contraception and SRH information and to highlight areas that continue to require further research.

\section{METHODS}

This systematic review evaluated studies relating to the post-abortion experiences of women living in lowand middle-income countries and their ability to access contraception and SRH information. Owing to the fact that PAC services are vital in all countries, even those where abortion laws are restrictive or prohibited, all studies situated in low- and middle-income countries have been considered for inclusion. ${ }^{7} 8$ Lowand middle-income counties and geographical regions have been defined using the Word Bank classification system for the 2016 fiscal year. ${ }^{10}$

The PICOS approach was used to develop the research question for this systematic review. ${ }^{11}$

- $P$ (refers to the patient, population or disease being addressed): in this review these were women who were obtaining or had obtained PAC services as well as PAC providers.

- I (relates to the intervention or exposure): in this case access to PAC services - specifically contraception and SRH information.

- $C$ (is the comparator group): the reported lack of access to PAC services in this review.

- $\mathrm{O}$ (refers to the outcome): adequate access to contraception and SRH information post-abortion.

- $S$ (the study design): qualitative, quantitative and mixedmethod studies were included in this review.

The research question the review sought to address was:

Does adequate access to post-abortion contraception and SRH increase uptake of contraception and SRH information in low- and middle-income counties?

Based on the Assessing the Risk of Bias of Individual Studies in Systematic Reviews of Healthcare Interventions guidelines, ${ }^{12}$ the methods used for assessing risk of bias in the articles selected for review included:

1 Checking the internal validity or conduct of the studies

2 The external validity or applicability of the studies

3 Study design

4 The reporting of results

5 Fidelity of intervention if any

6 Choice of outcome measures

7 Conflict of interest reported.

\section{Information sources}

Using the PRISMA Guidelines, Flow Diagram and Checklist, a systematic literature search was conducted by the first author from April to November 2014, a second search carried out in May 2015, and a final search conducted in June 2016 in order to identify new papers. ${ }^{11}$ Databases accessed in the search were: Ovid (MEDLINE), ProQuest, Science Direct, Web of Science, PUBMED and CINAHL, with additional articles sourced from the authors' records. References for the review were managed by the bibliographic software, Endnote X7, and a standard form was used to assist in data extraction. ${ }^{13}{ }^{14}$ Online supplementary Appendix 1 highlights the strategy used for the Ovid (MEDLINE) database search, which was used as a framework for subsequent database searches.

\section{Study selection}

Access to contraception within the context of this study relates to access to a wide range of contraceptive methods with the ability for a woman to make an informed decision, based on detailed and accurate information, regarding the method she feels would suit her best. ${ }^{15}{ }^{16} \mathrm{SRH}$ information is a broad term and has been used to encompass the provision of information, education and counselling relating to: reproductive health, such as fertility return, fertility intention, child-spacing, prevention 
of unwanted pregnancies, contraception methods and contraception use; sexual health advice, including information on signs of post-abortion complications and normal post-abortion symptoms, return to sexual activity advice, hygiene, sexually transmitted infection (STI)/HIV prevention, testing and treatment information; and information and/or referral to relevant and quality health services if needed. ${ }^{7} 1517$

The following search terms were used in combination to guide the study: post-abortion; post-abortion care; contraception; family planning; sexual and reproductive health information; sexual and reproductive health and rights; and comprehensive abortion care. Searches were restricted to English language only papers published between 2000 and 2016, and restricted to peer-reviewed journal articles through database filters.
Data collection, analysis, extraction and assessment

An initial 164 articles were identified by the first author from the six databases and an additional 36 articles from the authors' personal files were added to the review for a combined total of 200 articles. After removal of duplicates, the remaining 168 papers were assessed based on Title with a resulting 114 articles removed as they did not meet the inclusion/exclusion criteria. Manuscripts without abstracts were excluded as they were non-research papers. Two articles were not retrievable; however, on further review of their Title and Abstract information it was decided that the papers were not relevant to the study as their reported findings did not specifically relate to the research question. Some 52 full-text papers relevant to the review were assessed based on Title and Abstract information, leading to the inclusion of 19 papers for full article review and data extraction. ${ }^{18-35}$ Figure 1 presents the

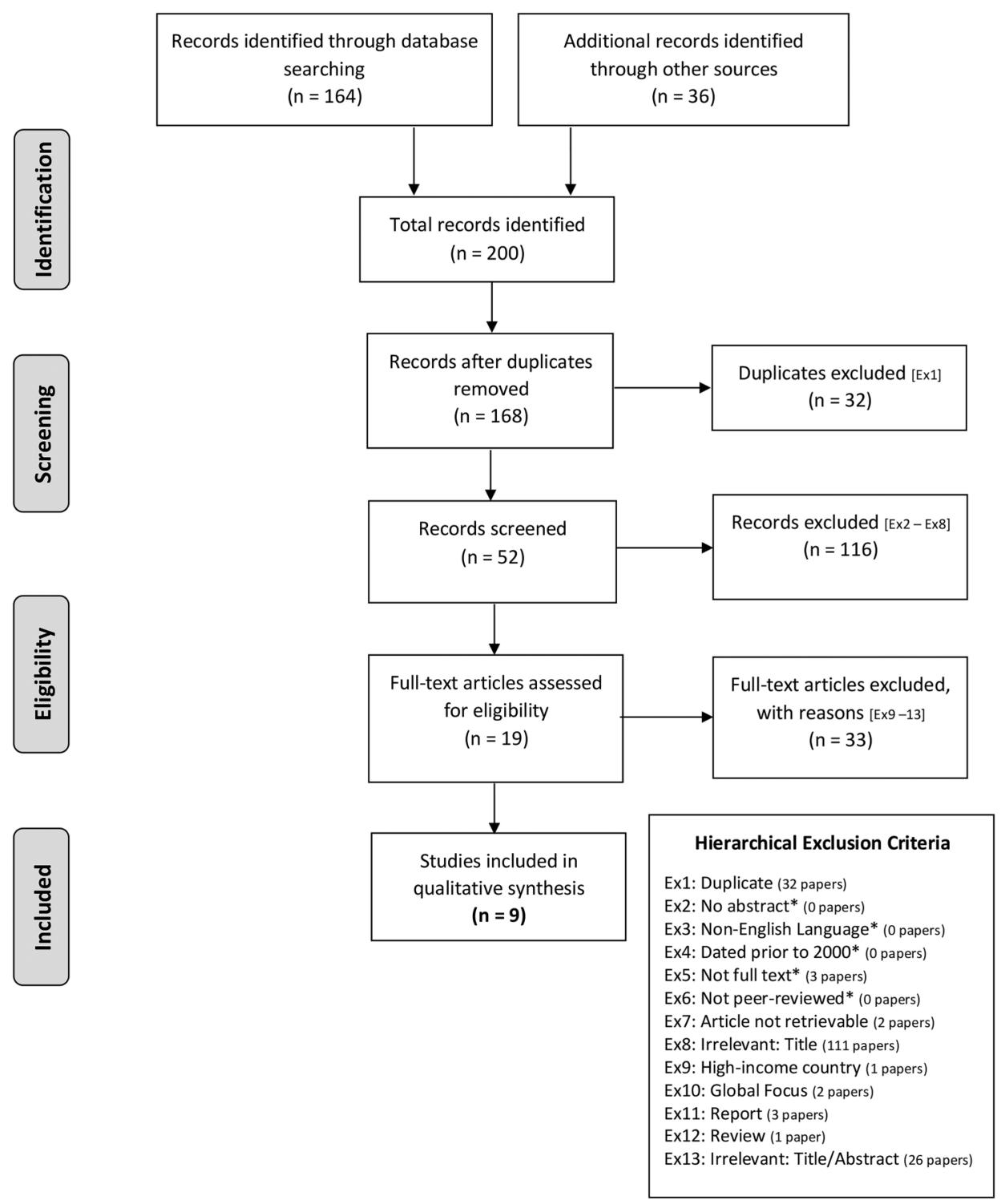

Figure 1 Systematic framework of the literature review process ${ }^{11}$ ( ${ }^{*}$ when possible, selected as filters during initial database searches). 
PRISMA Flow Diagram relating to this study and hierarchical exclusion criteria for article selection.

Both authors reviewed data extraction and synthesis for the final 19 articles selected for potential inclusion as well as the final nine selected papers. Online supplementary Appendix 2 displays the data extraction and synthesis of the 19 articles with potentiality for inclusion in the systematic review. Each of the 19 articles was examined to identify the role contraception access and the provision of SRH information for women post-abortion had within the study design and findings. Analysis of the articles involved the extraction and synthesis of relevant data into a standard form that was reviewed by both authors. As well as the detailed exclusion criteria, all articles also underwent quality assessment relating to the relevance of the study, the appropriateness of the research design and methodology, ethical considerations, and the journals in which the articles were published. ${ }^{14}$

\section{RESULTS}

After detailed appraisal, nine studies in total were selected for inclusion in the systematic review and are presented in Table $1 .{ }^{19} 2123-25$ 29-31 35 These studies were undertaken in four different geographical regions and within seven different countries. While there were varying legal implications with regards to obtaining an abortion within the study settings, all nine studies were conducted within PAC services or facilities. These included 94 government/public facilities (63 hospitals and 31 clinics); four nongovernmental facilities (two hospitals and two clinics); and one privately owned medical clinic.

\section{Study characteristics}

The review highlighted that studies did not specifically address access to contraception and SRH information post-abortion as the primary topic of research, the studies all discussed components of contraception access and uptake and the provision of various facets of SRH information to women post-abortion and were therefore selected for review. While studies concentrating on PAC provision for spontaneous abortion (miscarriage) and term unintended pregnancies (women admitted for delivery of an unintended pregnancy carried to term) were excluded from selection, one study included women from these two sets as control groups to compare with women seeking PAC services after unsafe abortion, and was therefore included. ${ }^{19}$

Participants in the nine studies were:

1 Women who were obtaining or had obtained PAC services only. ${ }^{19} 212430$

2 Women who were obtaining or had obtained PAC services as well as PAC providers (including medical staff, doctors, nurses and healthcare workers). ${ }^{23} 25293135$

No PAC provider-only studies were included. ${ }^{11} 14$ Three of the nine studies focused on post-abortion family planning services/contraception provision postabortion $^{21} 2429$ and six studies focused on PAC as a whole, ${ }^{19} 2325303135$ two of which incorporated generational aspects in their research. ${ }^{25} 30$

The research methodology employed in the articles included three quantitative studies; ${ }^{19} 2124$ three qualitative studies; ${ }^{25} \quad 30 \quad 31$ and three mixed-methods studies. $^{23} 29 \quad 35$ Nguyễn et al. $^{23}$ and McCarraher et al. $^{25}$ were research evaluations of PAC services which were components of larger implementation specifically designed to increase CAC (the CAC Project and CONECTA project, respectively). In total, quantitative data was obtained from 4595 individuals and qualitative data was provided by 1116 individuals across eight countries within the nine studies selected for this systematic review.

\section{Narrative synthesis of article content}

Owing to the relatively small number of studies found with specific reference to SRH information and contraception provision post-abortion, as well as the heterogeneity of the studies reviewed, a narrative synthesis of relevant outcomes reported in the chosen studies is presented. ${ }^{14}$

\section{Access to contraception post-abortion}

Barriers to contraception access for women who have undergone abortion are multifaceted and far reaching. In resource-poor settings physical access to a range of contraceptive methods can often be the first inhibitor for access for women. ${ }^{21} 253135$ In their 2010 evaluation of PAC services in the Dominican Republic, McCarraher et al. found that contraception was not available to PAC clients in some of the PAC facilities, and one-quarter of the study facilities visited were out of stock of one or more contraceptive methods. Some $21 \%$ of older women (aged 20-35 years) and 11\% of adolescents (under 19 years of age) reported leaving the hospital without a contraceptive because the hospital did not have the type they wanted (the contraceptive methods were not specified). ${ }^{25}$ The lack of contraceptive method availability combined with an absence of comprehensive contraception information and counselling has been highlighted as a barrier to contraception access and uptake. 1921232529

In Nepal, Rocca et al. ${ }^{21}$ found that of the total sample population $(n=838)$, one-third of the participants received no information or education on contraception choices, with over half of the sample population leaving abortion facilities without an effective method of contraception. Inadequate time for counselling, patient overcrowding, space limitations and lack of privacy are obstacles in the provision of effective counselling on post-abortion contraception. ${ }^{19} 21$ These barriers are often compounded by lack of PAC provider training, insufficient knowledge of staff, and socioculturally insensitive communication 


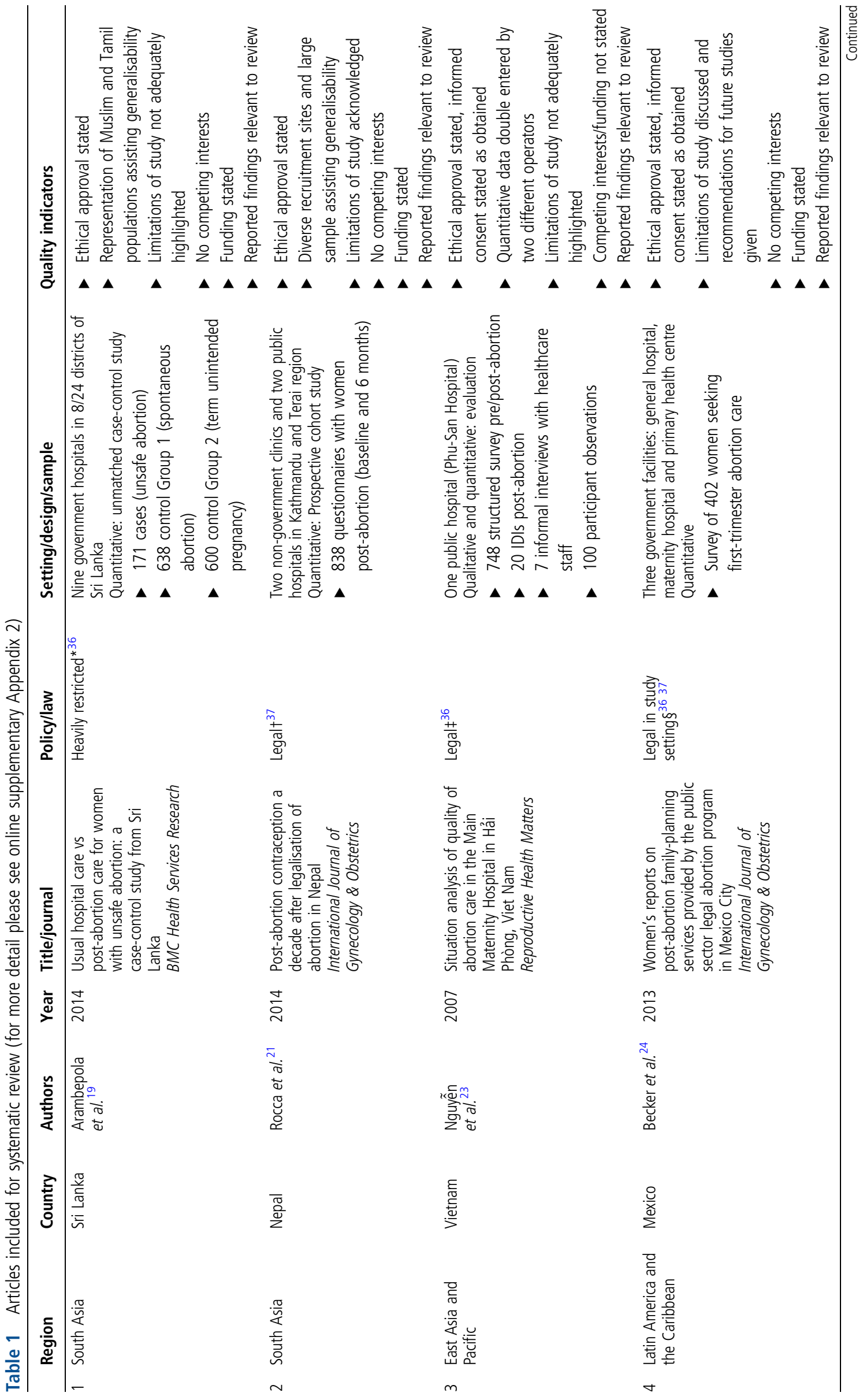




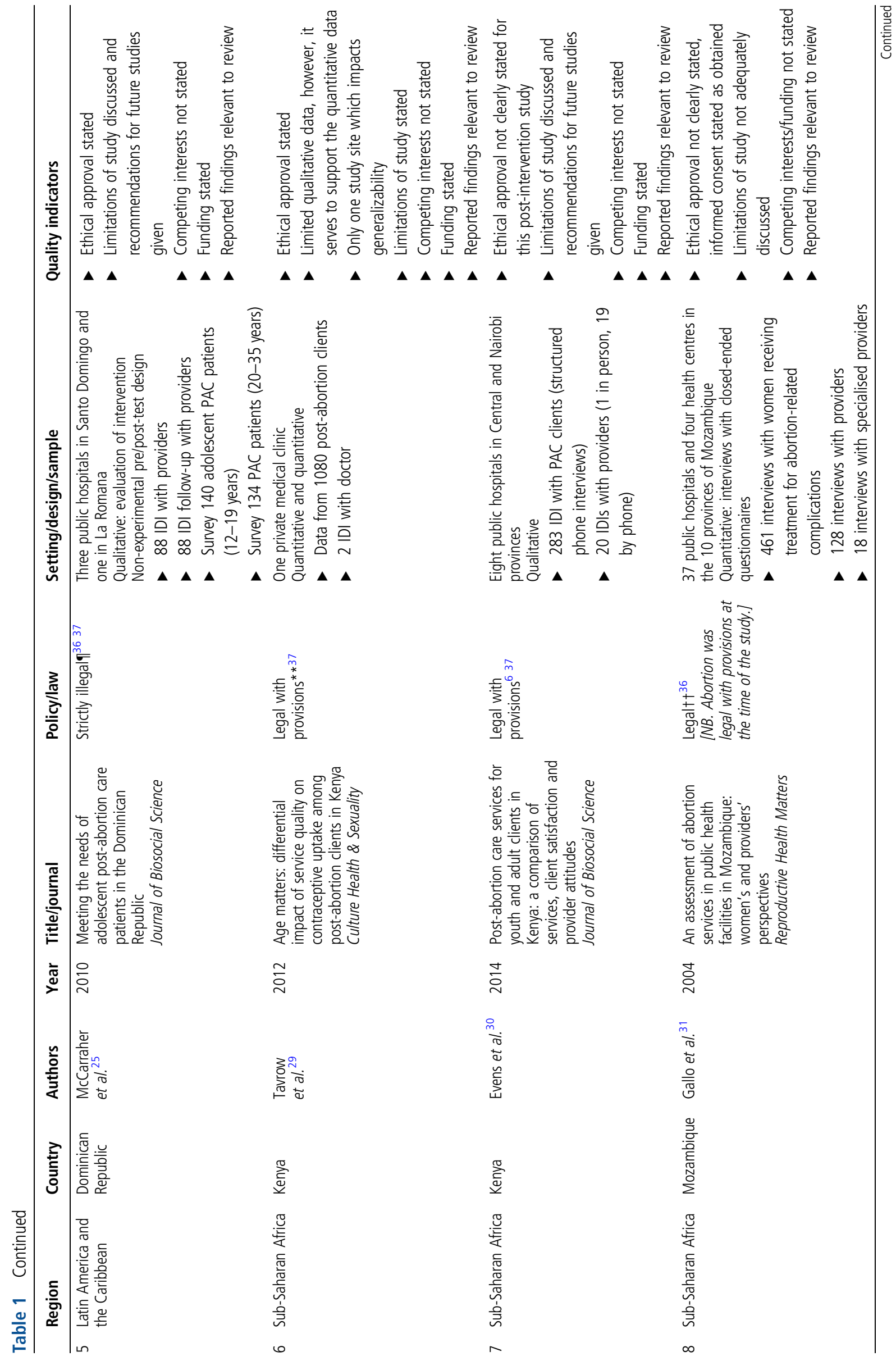




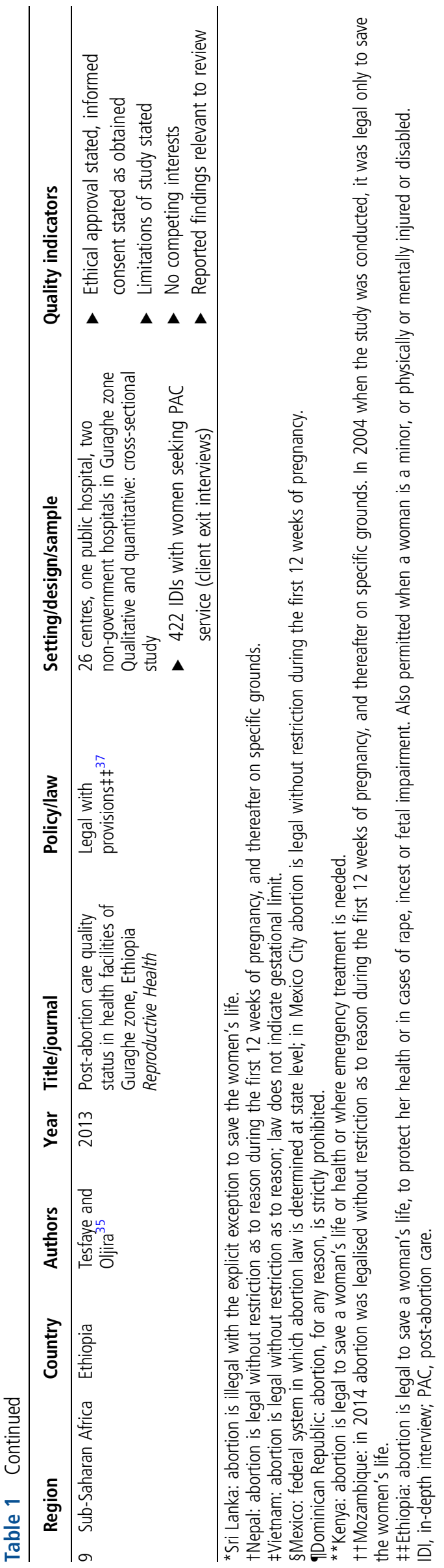

skills, which further impact access to information for women who need to make an informed decision regarding contraceptive use. ${ }^{19} 232430$ Rocca et al. ${ }^{21}$ also noted that only $19 \%$ of research participants received information on three or more contraceptive methods and approximately $31 \%$ received information on two methods, while two-thirds of women reported receiving information on at least one effective method of contraception. ${ }^{21}$ Lack of personalised contraceptive counselling, specifically relevant to the woman's life situation and previous contraceptive experiences, were also inhibitors to contraception uptake post-abortion. $^{23} 24$

Arambepola et al., ${ }^{19}$ in their Sir Lankan study, demonstrated that women who accessed in-patient PAC services at a large public hospital in Sri Lanka did not receive sufficient post-abortion access to contraception when compared with the study control groups (spontaneous abortion and term unintended pregnancies) and that contraceptive uptake and use was less at 6-8 weeks after hospital discharge. Attitudes of healthcare staff towards of women accessing PAC services, particularly in counties where abortion is illegal or restricted, contribute to the barriers women face when accessing contraception information and methods post-abortion. ${ }^{19}$ 23-25 35

The type of health service (i.e. government, nongovernment or private) has also been shown to have a direct link with post-abortion contraception access. Women utilising non-government or private facilities are more likely to receive information on contraceptive choices compared to women who attended public or government facilities. ${ }^{21} 2429$ The type of abortion procedure also shows correlation with lack of contraception access and uptake, with women accessing surgical abortions being more likely to receive information on, and access to, contraceptive methods compared to women having medical abortions. ${ }^{21} 24$

The reviewed studies also highlighted other barriers to access of contraceptive methods, information and education, such as: the gender of healthcare providers; ${ }^{24}$ if the woman's husband is away for extended periods; ${ }^{21}$ misconceptions regarding contraception; $;^{29}$ if a woman is not sexually active; ${ }^{24}$ or if the woman is an adolescent. 252930

\section{Access to SRH information post-abortion}

The provision of SRH information as an integral component of quality PAC services is often overlooked by service providers. Along with education regarding contraceptive options, discussing return to fertility is also an essential element of PAC. ${ }^{3}{ }^{7}$ Rocca et al. ${ }^{21}$ found that only half of their study sample population were informed about fertility return during their PAC visit, and in their Mexico City study Becker et al. ${ }^{24}$ reported $68 \%$ of their total research participants $(n=402)$ were educated on return to fertility information. 
In a study undertaken in the Dominican Republic in 2010, a high proportion of PAC service providers $(>70 \%)$ reported they routinely asked PAC patients about their fertility intentions and counselled them on contraception, STI/HIV and post-abortion complications. However, compared with provider reports, far fewer PAC patients indicated they had received counselling and information on risk of pregnancy, fertility intentions, STI/HIV risk, contraception availability and post-abortion complication. ${ }^{25}$ Similar discrepancies between provider and patient reporting was also detailed in a 2014 Kenyan study and 2004 study in Mozambique. ${ }^{31}$ When asked about information provision, just over half of the participants in Evens et al.'s ${ }^{30}$ study reported their provider had discussed return to fertility, HIV/STI information and testing, or provided information and access to contraception. In contrast, the vast majority of providers reported they routinely provide these services.

All studies reviewed revealed that post-abortion access to SRH information regarding SRH concerns and issues were inconsistently conveyed by PAC providers, if indeed at all. Gallo et al. ${ }^{31}$ highlight that PAC clients in Mozambique have been shown to have high STI rates, yet few of their research participants reported receiving condoms or information regarding their sexual health and STI/HIV testing, treatment and prevention. Several studies also documented lack of information provision on important SRH issues including: fertility return and intention; child-spacing; preventing unwanted pregnancies; contraceptive methods and use; information on emergency contraception; information on danger signs of post-abortion complications and normal post-abortion symptoms; return to sexual activity advice; and post-procedure hygiene. ${ }^{212425303135}$

\section{DISCUSSION}

A number of significant and intersecting themes concerning inhibitors to access of contraception and SRH information post-abortion emerged in the narrative content synthesis of the review. These include: lack of comprehensive information and education on a broad choice of contraception methods; insufficient commodity supply; provider attitudes; the type of service provider (government/public, nongovernment, private); as well as lack of effective and consistent SRH information and education provision to women post-abortion.

Similar to findings documented in several of the papers in review, ${ }^{19} 2124$ a six country United States Agency for International Development (USAID) study on interventions to strengthen contraceptive counselling and services also found that with effective contraceptive counselling, there is a marked increase in the number of women accepting contraception postabortion. ${ }^{2}{ }^{38}$ However, as McCarraher et al. ${ }^{25}$ state, improved contraceptive counselling is only one strategy to increase contraceptive uptake; availability of a broad range of contraceptive methods through consistent and effective commodity supply is fundamental for women in accessing their contraceptive method of choice post-abortion.

Judgmental (or perceived judgmental) provider attitudes have been shown to create barriers to access of contraception and SRH information post-abortion. Abortion-related stigma stems from the challenges abortion presents to social, cultural and religious beliefs. ${ }^{39} 40$ This stigma permits myths about abortion to propagate, can lead to shame and harassment and, particularly in countries where abortion is illegal or restricted, can be a barrier to women accessing highquality PAC services. ${ }^{2} 39{ }^{40}$ Inconsistencies in service provision across various provider facilities (government/public, non-government and private) has also been found to create barriers to access to contraception and SRH information post-abortion. While private facilities may offer comprehensive PAC, their provider fees deter women from accessing services. Alternatively, services provided (often free of charge) from government facilities lack the human resources to effectively provide adequate time to clients and may lack trained PAC providers.

Throughout the literature, the provision of SRH information in the form of counselling is inconsistently described. Counselling has been used to describe the provision of information and education specifically on contraception, while at other times the term has been used to describe more comprehensive provision of SRH information, closely related to the PAC Consortium definition of the term. ${ }^{67}$ While all nine studies emphasised issues relating to the provision of contraception and contraception counselling in PAC, no papers comprehensively addressed the provision of SRH information to PAC clients. However, five of the nine papers investigated components of SRH information (other than contraception information). This information related to: return to fertility or fertility intentions; ${ }^{21} 25 \quad 30 \quad 31$ STI/HIV information and/or testing; ${ }^{24} 25$ post-abortion complications; ${ }^{24} 25 \quad 3135$ and emergency contraception. ${ }^{24}$ The paucity of literature specifically relating to the provision of SRH information to women post-abortion is testament to the need for greater research on this topic.

\section{Limitations}

Lack of generalisability was a consistent limitation within all the reviewed studies, with several studies highlighting sampling and data collection difficulties such as participant recruitment and sample size as impacting the ability for generalisation to the wider population. ${ }^{19} 242930$ The use of self-reporting questionnaires within several of the studies has the potential to create social desirability and response bias. $^{19} 23 \quad 2433 \quad 35$ Interviewer and response bias may also play a role within the qualitative aspects of 
several of the studies. $\begin{array}{llllll}23 & 25 & 29 & 30 & 33 & \text { The sensitive }\end{array}$ nature of the topic and sociocultural beliefs regarding abortion may have been limiting factors within these studies, however, none of the papers reviewed addressed this issue adequately. The review was also restricted to articles published in English, and only the first author performed the first round of screening. While every consideration has been given to the context, characteristics and quality of the studies appraised, as this systematic review reports on studies from various demographic regions and countries, with varying policy regarding the legality of access to abortion services, the findings of this review must be considered within these parameters.

\section{Recommendations}

This systematic review highlights the lack of current literature relating to women's access to contraception and SRH information post-abortion. While the findings reiterate much of the current understanding regarding the complexities surrounding women's access to contraception post-abortion, they also uniquely highlight the inconsistencies relating to what providers consider counselling and SRH provision in PAC services. ${ }^{7}$ Further research on the type and quality of SRH information provided during PAC counselling is urgently needed to determine the scope and consistency of counselling currently being provided. This information has the potential to inform detailed PAC counselling frameworks that can assist PAC providers to more effectively meet women's postabortion information and educational needs.

The review indicated that with access to a wide range of contraceptive methods together with comprehensive SRH information and education, contraception uptake in women post-abortion was shown to increase. However, inconsistency in effective service provision; judgmental (or perceived judgmental) attitude of service providers to patients; restricted access to services and comprehensive SRH information; and the lack of availability of a broad range of contraceptive choices, continue to inhibit women's access to contraception and SRH information, post-abortion. Further research is needed to examine and document these barriers to post-abortion contraception and SRH information and to highlight the need for effective and equitable PAC provision for women and girls in low- and middle-income countries.

\section{CONCLUSION}

Abortions continue to impact the health and lives of women and girls around the world whether legally allowed or restricted. This review highlights a critical need and that access to affordable, equitable and highquality PAC services reduces morbidity and mortality resulting from incomplete and unsafe abortion and post-abortion complications. Through effective and equitable PAC, timely access to contraceptive methods and comprehensive SRH information is a key factor in assisting women to space births, prevent unintended pregnancies, avert unsafe abortions, and support women to make informed decisions and take control of their SRH and rights.

Twitter Follow Claire Rogers @ClaireRogersttN and Jaya Dantas@JayaDantas

Acknowledgements The authors wish to thank the three anonymous reviewers who provided useful feedback, comments and recommendations for this article.

Contributors CR and JARD conceptualised the study, developed objectives, framework and search strategy. CR developed protocol, searched and reviewed articles within the designated framework and prepared the first draft. JARD verified the reviewed articles, draft manuscript and added contextual applications. CR and JARD read and mutually approved the final manuscript.

Competing interests None declared.

Provenance and peer review Not commissioned; externally peer reviewed.

\section{REFERENCES}

1 Singh S, Darroch JE, Ashford LS. Adding It Up: The Costs and Benefits of Investing in Sexual and Reproductive Health 2014. New York, NY: UNFPA and Guttmacher Institute, 2014.

2 Shah IH, Åhman E, Ortayli N. Access to safe abortion: progress and challenges since the 1994 International Conference on Population and Development (ICPD). Contraception 2014;90(6 Suppl.):S39-S48.

3 Barot S. Implementing postabortion care programs in the developing world: ongoing challenges. Guttmacher Policy Rev 2014;17:22-28.

4 Hyman A, Kumar A. A woman-centered model for comprehensive abortion care. Int J Gynaecol Obstet 2004;86:409-410.

5 Ipas. Comprehensive Abortion Care. Secondary Comprehensive Abortion Care. 2015. http://www.ipas.org/en/What-We-Do/ Comprehensive-Abortion-Care.aspx [accessed 2 January 2017].

6 Postabortion Care (PAC) Consortium. Service Delivery Task Force. Essential Elements of Postabortion Care: Service Delivery Barriers and Resources. PAC Consortium, 2014.

7 PAC Consortium Community Task Force. Essential Elements of Postabortion Care: An Expanded and Updated Model. Postabortion Care Consortium, 2002.

8 Rasch V. Unsafe abortion and postabortion care - an overview. Acta Obstet Gynecol Scand 2011;90:692-700.

9 Adinma E. Unsafe abortion and its ethical, sexual and reproductive rights implications. West Afr J Med 2011;30:245-249.

10 The World Bank Group. World Bank Country and Lending Groups. 2015. http://data.worldbank.org/about/ country-and-lending-groups [accessed 2 January 2017].

11 Liberati A, Altman DG, Tetzlaff J, et al. The PRISMA Statement for reporting systematic reviews and meta-analyses of studies that evaluate health care interventions: explanation and elaboration. PLoS Med 2009;6:e1000100.

12 Viswanathan M, Ansari MT, Berkman ND, et al. Assessing the Risk of Bias of Individual Studies in Systematic Reviews of Healthcare Interventions. Methods Guide for Comparative Effectiveness Reviews. Agency for Healthcare Research and Quality Methods Guide for Comparative Effectiveness Reviews. March 2012. 
13 Daly J, Willis K, Small R, et al. A hierarchy of evidence for assessing qualitative health research. J Clin Epidemiol 2007;60:43-49.

14 Harris JD, Quatman CE, Manring MM, et al. How to write a systematic review. Am J Sports Med 2014;42:2761-2768.

15 World Health Organization (WHO). Ensuring Human Rights in the Provision of Contraceptive Information and Services: Guidance and Recommendations. Geneva, Switzerland: WHO, 2014.

16 World Health Organization (WHO). Health Workers Roles in Providing Safe Abortion Care and Post-abortion Contraception. Geneva, Switzerland: WHO, 2015.

17 World Health Organization (WHO). Sexual Health, Human Rights and the Law. Geneva, Switzerland: WHO, 2015.

18 Acharya R, Kalyanwala S. Knowledge, attitudes, and practices of certified providers of medical abortion: evidence from Bihar and Maharashtra, India. Int J Gynaecol Obstet 2012;118 (Suppl. 1):S40-S46.

19 Arambepola C, Rajapaksa LC, Galwaduge C. Usual hospital care versus post-abortion care for women with unsafe abortion: a case control study from Sri Lanka. BMC Health Serv Res 2014;14:470.

20 Azmat SK, Shaikh BT, Mustafa G, et al. Delivering post-abortion care through a community-based reproductive health volunteer programme in Pakistan. J Biosoc Sci 2012;44:719-731.

21 Rocca CH, Puri M, Harper CC, et al. Postabortion contraception a decade after legalization of abortion in Nepal. Int J Gynaecol Obstet 2014;126:170-174.

22 Htay TT, Sauvarin J, Khan S. Integration of post-abortion care: the role of township medical officers and midwives in Myanmar. Reprod Health Matters 2003;11:27-36.

23 Nguyễn $\mathrm{MH}$, Gammeltoft $\mathrm{T}$, Rasch V. Situation analysis of quality of abortion care in the main maternity hospital in Hải Phòng, Viet Nam. Reprod Health Matters 2007;15:172-182.

24 Becker D, Díaz Olavarrieta C, Garcia SG, et al. Women's reports on postabortion family-planning services provided by the public-sector legal abortion program in Mexico City. Int J Gynaecol Obstet 2013;121:149-153.

25 McCarraher DR, Chen-Mok M, Oronoz AS, et al. Meeting the needs of adolescent post-abortion care patients in the Dominican Republic. J Biosoc Sci 2010;42:493-509.

26 Esber A, Foraker RE, Hemed M, et al. Partner approval and intention to use contraception among Zanzibari women presenting for post-abortion care. Contraception 2014;90:23-28.

27 Etuk SJ, Ebong IF, Okonofua FE. Knowledge, attitude and practice of private medical practitioners in Calabar towards post-abortion care. Afr J Reprod Health 2003;7:55-64.
28 Kalu CA, Umeora OUJ, Sunday-Adeoye I. Experiences with provision of post-abortion care in a university teaching hospital in south-east Nigeria: a five year review. Afr J Reprod Health 2012;16:105-112.

29 Tavrow P, Withers M, McMullen K. Age matters: differential impact of service quality on contraceptive uptake among post-abortion clients in Kenya. Cult Health Sex 2012;14:849-862.

30 Evens E, Otieno-Masaba R, Eichleay M, et al. Post-abortion care services for youth and adult clients in Kenya: a comparison of services, client satisfaction and provider attitudes. J Biosoc Sci 2014;46:1-15.

31 Gallo MF, Gebreselassie H, Victorino MTA, et al. An assessment of abortion services in public health facilities in Mozambique: women's and providers' perspectives. Reprod Health Matters 2004;12(24, Suppl.):218-226.

32 Jackson E, Johnson BR, Gebreselassie H, et al. A strategic assessment of unsafe abortion in Malawi. Reprod Health Matters 2011;19:133-143.

33 Kinaro J, Mohamed Ali TE, Schlangen R, et al. Unsafe abortion and abortion care in Khartoum, Sudan. Reprod Health Matters 2009;17:71-77.

34 Paul M, Gemzell-Danielsson K, Kiggundu C, et al. Barriers and facilitators in the provision of post-abortion care at district level in central Uganda - a qualitative study focusing on task sharing between physicians and midwives. BMC Health Serv Res 2014;14:28.

35 Tesfaye G, Oljira L. Post abortion care quality status in health facilities of Guraghe zone, Ethiopia. Reprod Health 2013;10:35.

36 Center for Reproductive Rights. The World's Abortion Laws 2014. New York, NY: Center for Reproductive Rights, 2014.

37 Center for Reproductive Rights. Abortion Worldwide: Seventeen Years of Reform. New York, NY: Center for Reproductive Rights, 2011.

38 High Impact Practices: Family Planning. Postabortion Family Planning: Strengthening the family planning component of postabortion care. 2012. https://www.fphighimpactpractices. org/resources/postabortion-family-planning-strengtheningfamily-planning-component-postabortion-care [accessed 2 January 2017].

39 Gold J, Hurley L, Wachsmann H, et al. How to Talk about Abortion: A Guide to Rights-based Messaging. London, UK: International Planned Parenthood Foundation (IPPF), 2015.

40 Hanschmidt F, Linde K, Hilbert A, et al. Abortion stigma: a systematic review. Perspect Sex Reprod Health 2016;48:169-177. 